\title{
A Evaluation on Removing of Rain from Images
}

\author{
R.Sunanda, J.Beatrice Seventline
}

\begin{abstract}
In this paper, different techniques for the detection and removal of rain from images have been reviewed. The performance of each technique varies as the rain reduces the visibility of the scene in the image. Detection and removal of rain needs the discrimination of rain from non-rain pixels. Accuracy of methods depends upon this discrimination. Here merits and demerits of existing methods are discussed, which motivates further research. A rain removal technique has wide applications in indoor and outdoor security surveillance systems, tracking and navigation, entertainment industries and consumer electronics.
\end{abstract}

Keywords: Rain streaks, Guided filter, Dictionary learning, Sparse coding, Image decomposition, Neural network.

\section{INTRODUCTION}

Distortions in still images caused by bad weather conditions can have an ocular negative affect on the performance of many visual systems. Rain comes under one of inclemency conditions, which causes significant variations in images. A study by Garg [1] reveals that inclemency conditions, i.e., rain and snow belong to the dynamic weather. This dynamic weather includes constituent particles of relatively large sizes that are caught by cameras. Steady weather, i.e., haze particles are smaller in size and can hardly be caught. So, rain or snow contributes complex pixel variations and beclouds the information that is brought in the image or video.

The rain and snow have some differences when looking at the image or video. First, the rain is semi- transparent and because of this, the objects in the image or video will have blur and the information is lost in the background edges. Second, the pixels with different intensities will be affected by rain differently. The rain will enhance its intensity if the pixel's primary intensity is relatively low. When a high intensity pixel is subjected to rain, its intensity will become lower. So, rain corrupted pixels in an image will have the same intensity because the reflection of rain is dominating under this scenario. Snow is untransparent and can largely becloud the object. In addition to this, snow has bright and white color, and snow's reflection is stronger than rain. This leads to high intensity values in an image caused by the snow.

In literature, the rain removal is divided into two step problem. One is detecting rain affected pixels. Second is removing of rain affected pixels captured. Rain detection identifies the location of damaged pixels caused by rain. Based on the temporal properties of rain, the rain drops will not affect the rainy pixel but also damage the neighboring pixels. Rain removal method removes the detected rain

Revised Manuscript Received on August 14, 2019.

R. Sunanda, Dept of ECE, GITAM University, Visakhapatnam, Andhra Pradesh, India (Email: sunanda443@gmail.com)

Dr. J. Beatrice Seventline, Dept of ECE, GITAM University, Visakhapatnam, Andhra Pradesh , India (Email: seventline.joseph@gitam.edu) pixels from the image. Rain removal techniques can be applied to rain affected images. According to Tripathi \& Mukhopadhyay (2014) [20], rain removal techniques were classified into two main approaches, which are time domain based approach and frequency domain based approach. In the time domain based approach basically considered the properties of rain itself, including the chromatic, temporal or both.

This paper is arranged as follows. Rain properties and appearance are analyzed in Section II. Literature review is presented in Section III. Conclusion is presented in Section IV.

\section{RAIN ANALYSIS}

Rain streaks in the image which is caused by bad weather conditions, which has two integral properties in still images. They are spatial and chromatic properties.

\section{Spatial}

Rain appears as a collection of spherical water droplets. These droplets are distributed randomly in space. Due to the gravitational force, the rain drops fall at high speed when they are near the ground and might not seen in successive frames [1]. The affect of rain drops are similar to haze or fog, when they are far away from the camera. Rain distributes in every image randomly. The pixels which are damaged by rain can have similar intensity distribution [1]. The intensity variations are almost similar for the adjacent pixels with similar background. For the pixels which are never subjected to rain, their intensity distribution follows uniform distribution without any movement of the objects captured in an image.

\section{Chromatic}

A stationary rain drop appears like a transparent sphere and refracts light in a wider optical field. The surface and the inner reflections make the pixel subjected to rain much brighter than its background. The intensities are approximately the same even though the background intensities are very different [1]. The edges of the object in the background of an image to be preserved. As the chromatic properties would not depend on velocity and direction of rain streaks it is used to remove the blur affect of rain pixel location in an image.

\section{LITERATURE REVIEW}

Distortions in still images caused by bad weather conditions can have an ocular negative affect on the 
performance of many visual systems. Detecting and removing the rain from single frame was proposed in [2], where the authors used image decomposition using bilateral filter, sparse coding and Morphological Component Analysis (MCA) based dictionary learning algorithms. The same authors in [3][17], extended the work in [2] to image/video by introducing automatically self learning the dictionaries. The authors first decomposed an image into the Low Frequency (LF) and High Frequency (HF) parts using a bilateral filter. The HF part was again decomposed into a "rain component" and a "non-rain component" by using dictionary learning and sparse coding. No extra training samples were required in the dictionary learning for image de-noising. The authors in [4] used context constrained image segmentation to a rain image to divide patches with different context categories and achieved self learning of rain patterns by utilizing PCA and SVM classifiers. But the disadvantage is that lost details and complexity in time. In this work, PSNR metric is considered as quantitative performance. The PSNR parameter is tabulated in Table II. The PSNR of [4] is better than Bilateral, K-SVD and MCA by $4.5 \%, 0.66 \%$ and $0.09 \%$ respectively for figure 4 . The PSNR of [4] performs better than Bilateral, K-SVD and MCA by $21.58 \%, 19.68 \%$ and $0.41 \%$ respectively for figure 5. Single color image based rain removal framework was proposed in [5], where a color image is decomposed into LF and $\mathrm{HF}$ by using guided image filtering. HF is further decomposed into rain and non-rain components using dictionary learning and sparse coding. Histogram of oriented Gradients (HoG), Depth of Field (DoF) and Eigen Color (EC) are employed to decompose the HF to separate rain streaks from the image. In this work, Visual Information Fidelity (VIF) metric is considered as quantitative performance. The VIF parameter is tabulated in Table III. The VIF performs of [5] better than Nonlocal, Bilateral, KSVD and MCA by $78 \%, 58 \%, 18 \%$ and $0 \%$ respectively for figure 4. The VIF of [5] is better than Nonlocal, Bilateral, K-SVD and MCA by $58.69 \%, 28.26 \%, 26.08 \%$ and $2.17 \%$ respectively for figure 5 . VIF outperforms than PSNR. The authors in [1]-[5] have applied Fig.1 and removed rain from images. The dictionary learning is time taking process where the noise is included at the input side has less information at the location of rain streaks in an image.

An algorithm is proposed in [6], in which the authors considered the common characteristics of rain and snow caused by the bad weather conditions. Towards this, the authors developed Principal Direction of an Image Patch (PDIP) and the Sensitivity of Variance of Color Channel (SVCC) to describe the difference of rain or snow from other image components. A guided filter is used to decompose an image. For HF part, dictionary learning and three classifications of dictionary atoms are implemented to decompose it into non-dynamic components and dynamic (rain or snow) components as shown in Fig.2.

A guided filter is used in [7] to remove rain and snow. A refined guidance image method [8] is considered to separate rain and snow in a single image. This method is also suitable for both rain and snow removal better than guide filter. Without the need of rain pixel detection, a generalized

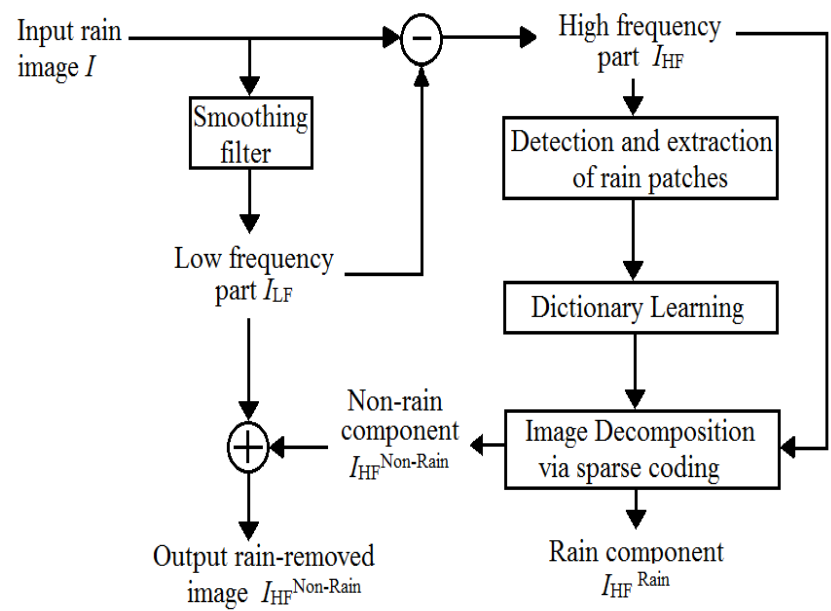

Fig 1. Rain removal framework

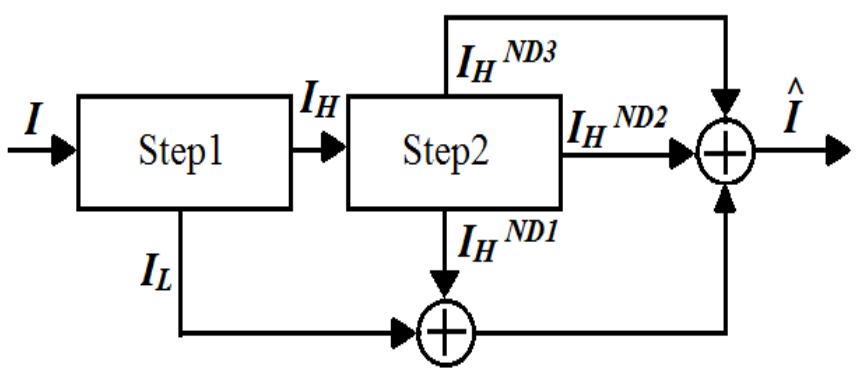

Fig. 2. The simplified pipeline of algorithm [6]

low rank appearance model is proposed by the author in [9], in which rain streaks patterns are considered. This model characterizes the spatio temporally correlated rain streaks, and thus could tackle both image and video rain removal at one instance. This model decomposes the rainy image into rain streak component, rain-free component, and imaging noise. This model is not limited to any particular source input. The model does not require pre-processing and time consuming dictionary learning. $\mathrm{L}_{0}$ gradient minimization approach [10] is proposed to remove the rain pixels. The minimization technique globally controls how many nonzero gradients are resulted in an image. The method locates important edges globally. These edges are preserved and low amplitude and insignificant details are diminished. In this way the rain pixels are removed.

Discriminative sparse coding based approach [11] for single image de-raining considers a non linear screen blend model for modeling rain images. By learning a dictionary with mutual exclusivity, the de rained image layer and the rain layer can be accurately separated using sparse coding with high discriminability. The ambiguities between derained and rained layers in low pass channels leads to some artifacts around rain streaks. This method does not work when image has many structures similar to rain drops and water drops stick to the glasses. 


\section{RESULTS \& DISCUSSIONS}

Table I.

Comparison of previous works on rain removal

The proposed method in [12] formulates a global sparse model that involves three sparse terms by considering the intrinsic

\begin{tabular}{|c|c|}
\hline Papers & Methods \\
\hline Garg et al. [1] & $\begin{array}{l}\text { Temporal properties for } \\
\text { detection and photometric } \\
\text { constraints for refinement }\end{array}$ \\
\hline Fu and Kang et al. [2] & $\begin{array}{l}\text { Image decomposition } \\
\text { dictionary learning and sparse } \\
\text { coding for identifying rain } \\
\text { components }\end{array}$ \\
\hline $\begin{array}{l}\text { Kang and Lin et al. } \\
\text { [3] }\end{array}$ & $\begin{array}{l}\text { Image decomposition } \\
\text {,automatic dictionary learning } \\
\text { and sparse coding for } \\
\text { identifying rain components }\end{array}$ \\
\hline Haung et al. [4] & $\begin{array}{l}\text { Context constrained image } \\
\text { segmentation, Image } \\
\text { decomposition, dictionary } \\
\text { learning and sparse coding for } \\
\text { identifying rain components }\end{array}$ \\
\hline Chen et al. [5] & $\begin{array}{l}\text { Image decomposition } \\
\text { dictionary learning, depth of } \\
\text { field, Eigen color, height of } \\
\text { oriented gradients and sparse } \\
\text { coding for identifying rain } \\
\text { components in color image }\end{array}$ \\
\hline tal. [6] & $\begin{array}{l}\text { common characteristics of rain } \\
\text { and snow, Image decomposition } \\
\text {, dictionary learning , PDIP, } \\
\text { SVCC and for identifying rain } \\
\text { components }\end{array}$ \\
\hline Jing $\mathrm{Xu}$ et al. [7] & $\begin{array}{l}\text { Guidance image and guided } \\
\text { filter to remove rain and snow }\end{array}$ \\
\hline $\mathrm{Xu}$ et al. [8] & $\begin{array}{l}\text { Refined Guidance image and } \\
\text { guided filter to remove rain and } \\
\text { snow }\end{array}$ \\
\hline Hsu et al. [9] & $\begin{array}{l}\text { Spatio-temporal properties for } \\
\text { removing rain streaks in image } \\
\text { and video }\end{array}$ \\
\hline Manu [10] & $\begin{array}{l}\mathrm{L}_{0} \text { gradient minimization foe } \\
\text { removing rain pixels }\end{array}$ \\
\hline Luo et al. [11] & $\begin{array}{l}\text { Non linear screen blend model } \\
\text { and discriminative sparse } \\
\text { coding for removing rain pixels }\end{array}$ \\
\hline Deng et al. [12] & $\begin{array}{l}\text { Alternating direction method of } \\
\text { multipliers }\end{array}$ \\
\hline Shen et al. [13] & $\begin{array}{l}\text { CNN based wavelet and dark } \\
\text { channel }\end{array}$ \\
\hline Chen et al. [14] & $\begin{array}{l}\text { Linear additive composite } \\
\text { model and screen blend model }\end{array}$ \\
\hline
\end{tabular}

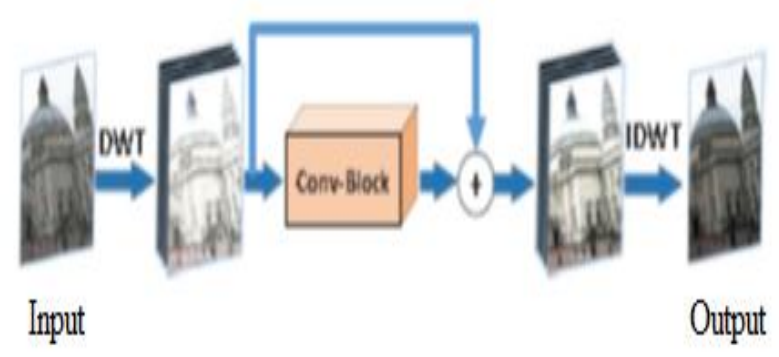

Fig. 3: Rain removal network architecture using CNN $[13],[14]$

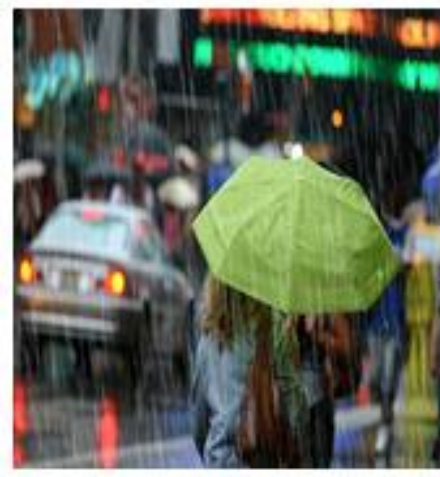

Fig. 4

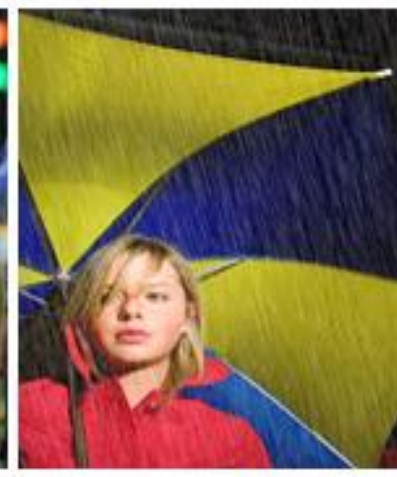

Fig. 5 directional and structural knowledge of rain streaks, as well as the property of image background information. The authors employed alternating direction method of multipliers (ADMM) to solve the proposed convex model which guarantees the global optimal solution. Convolutional Neural Network (CNN) based on wavelet and dark channel was proposed in [13]. Rain streaks near to the lens appear as noise in an image, whereas the rain from distance appears as haze veil. The authors combined above two separate structures to one network, the final model was developed as an end to end structure for rain and haze removal. The authors in [14] considered Linear Additive Composite Model (LACM) and Screen Blend Model (SCM model) and developed a Customized Filter Rain Detection and Removal Network (CRDRN) to detect and remove the rain streaks from an image. Comparison of previous works on rain removal with methods is tabulated below.

\section{Table II}

Performance comparisons in psnr (db) using bilateral filtering [15], k-svd based image denosing [16], mca based method [17], method in [4]

\begin{tabular}{|l|l|l|l|l|}
\hline & $\begin{array}{l}\text { Bilateral } \\
{[15]}\end{array}$ & $\begin{array}{l}\text { K-SVD } \\
{[16]}\end{array}$ & $\begin{array}{l}\text { MCA } \\
{[17]}\end{array}$ & {$[4]$} \\
\hline Figure 4 & 20.08 & 20.90 & 21.02 & 21.04 \\
\hline Figure 5 & 19.00 & 19.46 & 24.13 & 24.23 \\
\hline
\end{tabular}




\section{Table III}

Performance comparisons in vif using bilateral filtering [15], k-svd [18], non local [19], mca based method [17], method in [5]

\begin{tabular}{|c|c|c|c|c|c|}
\hline & $\begin{array}{c}\text { Nonlo } \\
\text { cal [19] }\end{array}$ & $\begin{array}{c}\text { Bilate } \\
\text { ral [15] }\end{array}$ & $\begin{array}{c}\text { K- } \\
\text { SVD } \\
{[16]}\end{array}$ & $\begin{array}{c}\text { MC } \\
\text { A [17] }\end{array}$ & [5] \\
\hline $\begin{array}{c}\text { Figur } \\
\text { e 4 }\end{array}$ & 0.11 & 0.21 & 0.41 & 0.50 & \multicolumn{2}{|c|}{0.5} \\
\hline $\begin{array}{c}\text { Figur } \\
\text { e 5 }\end{array}$ & 0.19 & 0.33 & 0.34 & 0.45 & \multicolumn{2}{|c|}{0.4} \\
\hline
\end{tabular}

\section{CONCLUSION}

Distortions in still images caused by bad weather conditions can have an ocular negative affect on the performance of many visual systems. Removal of rain from images is one of the key issues as the rain removed image gets blurred. In this paper, the rain detection and removal techniques used by researchers have been discussed. The detection and removal of rain is challenging because rain has spatial and temporal properties which can affect the neighboring pixels in an image. Therefore, our research direction will raise the issues on the detection of rain in a single color image

\section{REFERENCES}

1. K. Garg and S. K. Nayar, "Detection and removal of rain from videos," Proceedings of the 2004 IEEE Computer Society Conference on Computer Vision and Pattern Recognition, 2004. CVPR 2004., Washington, DC, USA, 2004, pp. I-I.

2. Y. Fu, L. Kang, C. Lin and C. Hsu, "Single-frame-based rain removal via image decomposition," 2011 IEEE International Conference on Acoustics, Speech and Signal Processing (ICASSP), Prague, 2011, pp. 14531456.

3. L. Kang, C. Lin, C. Lin and Y. Lin, "Self-learning-based rain streak removal for image/video," 2012 IEEE International Symposium on Circuits and Systems, Seoul, 2012, pp. 1871-1874.

4. D. Huang, L. Kang, M. Yang, C. Lin and Y. F. Wang, "Context-Aware Single Image Rain Removal," 2012 IEEE International Conference on Multimedia and Expo, Melbourne, VIC, 2012, pp. 164-169.

5. D. Chen, C. Chen and L. Kang, "Visual Depth Guided Color Image Rain Streaks Removal Using Sparse Coding," in IEEE Transactions on Circuits and Systems for Video Technology, vol. 24, no. 8, pp. 1430-1455, Aug. 2014.

6. Y. Wang, S. Liu, C. Chen and B. Zeng, "A Hierarchical Approach for Rain or Snow Removing in a Single Color Image," in IEEE Transactions on Image Processing, vol. 26, no. 8, pp. 3936-3950, Aug. 2017.

7. Jing Xu, Wei Zhao, Peng Liu \& Xianglong Tang, “An Improved Guidance Image Based Method to Remove Rain and Snow in a Single Image" in Computer and Information Science Vol. 5, No. 3; May 2012.

8. J. Xu, W. Zhao, P. Liu and X. Tang, "Removing rain and snow in a single image using guided filter," 2012 IEEE International Conference on Computer Science and Automation Engineering (CSAE), Zhangjiajie, 2012, pp. 304-307.

9. Y. Chen and C. Hsu, "A Generalized Low-Rank Appearance Model for Spatio-temporally Correlated
Rain Streaks," 2013 IEEE International Conference on Computer Vision, Sydney, NSW, 2013, pp. 1968-1975.

10. B. N. Manu, "Rain removal from still images using L0 gradient minimization technique," 2015 7th International Conference on Information Technology and Electrical Engineering (ICITEE), Chiang Mai, 2015, pp. 263-268.

11. Y. Luo, Y. Xu and H. Ji, "Removing Rain from a Single Image via Discriminative Sparse Coding," 2015 IEEE International Conference on Computer Vision (ICCV), Santiago, 2015, pp. 3397-3405.

12. Liang-Jian Deng, Ting-Zhu Huang, Xi-Le Zhao, TaiXiang Jiang, A directional global sparse model for single image rain removal, Applied Mathematical Modelling (2018).

13. Shen, Liang et al. "Deep joint rain and haze removal from single images." CoRRabs/1801.06769 (2018): n. pag.

14. Tianyi Chen and Chengzhou Fu "Single-image-based Rain Detection and Removal via CNN" J. Phys.: Conf. Ser. 1004012007

15. C. Tomasi and R. Manduchi, "Bilateral filtering for gray and color images," Proc. IEEE Int. Conf. Comput.Vis., Jan. 1998.

16. M. Aharon, M. Elad, and A. M. Brukstein,"The K-SVD: An algorithm for designing of overcomplete dictionaries for sparse representation," IEEE Trans. Signal Process., vol.54, no. 11, pp. 4311-4322, Nov. 2006.

17. L. W. Kang, C. W. Lin, and Y. H. Fu, "Automatic Single image-based rain streaks removal via image decomposition", IEEE Trans. Image Proc., vol. 21, no. 4,pp.1742-1755,2012.

18. M. Elad and M. Aharon, "Image denoising via sparse and redundant representations over learned dictionaries," IEEE Trans. Image Process., vol. 15, no. 12, pp. 37363745, Dec. 2006

19. A. Buades, B. Coll, and J.-M. Morel, "Nonlocal image and movie denoising," Int. J. Comput. Vis., vol. 76, no. 2 pp. 123-139, 2008

20. Tripathi, A.K. \& Mukhapadhyay, S.SIViP (2014) 8 1421. https://doi.org/10.1007/s11760-012-0373-6

\section{AUTHORS PROFILE}

R. Sunanda Dept of ECE, GITAM University, Visakhapatnam, India Email ID: sunanda443@ gmail.com

Dr. J. Beatrice Seventline Dept of ECE, GITAM University, Visakhapatnam, India

Email ID: seventline.joseph@gitam.edu 